\title{
Primary malignant melanoma of urinary bladder
}

\begin{abstract}
Primary malignant melanoma of urinary bladder is very rare. About 24 cases have been reported till date. A 53-year-old female presented with history of gross hematuria. Cystoscopy revealed blackish solitary papillary growth near bladder neck. Transurethral resection was performed, which showed pigmented and atypical melanocytes. Tumor was positive for S-100 and HMB-45. Clinical and radiological examinations showed no other focus of disease. Patient did not receive any further treatment, later presented with metastases. Received palliative radiation therapy to the painful spine metastases. She succumbed to widespread disease 14 months after the diagnosis.
\end{abstract}

Keywords: melanoma, urinary bladder
Volume 5 Issue 4 - 2017

\author{
Nagarjuna Burela,' Nidhi Patni, ${ }^{2}$ Anjali \\ Sharma ${ }^{3}$ \\ 1,2Department of Radiation Oncology, Bhagwan Mahaveer \\ Cancer Hospital \& Research Centre, India \\ ${ }^{3}$ Department of Pathology, Bhagwan Mahaveer Cancer Hospital \\ \& Research Centre, India
}

\begin{abstract}
Correspondence: Nagarjuna Burela, Senior Resident, Department of Radiation Oncology, Bhagwan Mahaveer Cancer Hospital \& Research Centre, Jaipur, Rajasthan, India, Tel +9| 961054155I,Email nagarjunaburela@gmail.com
\end{abstract}

Received: August 02, 2017 | Published: December 12, 2017

\section{Introduction}

Primary malignant melanoma of genitourinary tract is rare and accounts for only $0.2 \%$ of all melanomas. Urethra is the most common site of origin, although primary malignant melanoma of urinary bladder is extremely rare. ${ }^{1}$ Only 24 cases of primary malignant melanoma of urinary bladder are reported to date. ${ }^{2}$ Malignant melanomas of bladder are usually metastatic lesions, which originate from distant primary site rather than being a primary lesion. ${ }^{3}$ We report a case of primary malignant melanoma of urinary bladder.

\section{Case history}

A 53-year-old female presented with one-month history of gross hematuria and increased frequency of urination. There was no past history of calculus. She did not have any medical comorbidities. She underwent hysterectomy after last childbirth at the age of 40years. General physical examination and routine blood investigations were normal. CECT scan of whole abdomen showed $4.4 \times 3.7 \times 2 \mathrm{~cm}$ polypoid enhancing mass in urinary bladder with bladder wall thickening. Cystoscopy revealed blackish solitary papillary growth near bladder neck from 10 o'clock to 2 o' clock position. A transurethral resection of the lesion was performed including deep muscular layers of the bladder wall. The respected specimen examination showed pigmented melanocytes infiltrating sub epithelial tissue below the transitional epithelium (Figure 1). Margins of resection were free, however, base was involved. Atypical melanocytes were seen at the margins of the tumor. Immunohistochemical study showed that tumor cells were positive for S-100 Figure 2 and HMB-45 Figure 3. They were negative for cytokeratin and SMA. Dermatologic, ophthalmologic, otorhinolaryngologic and proctologic evaluations were negative. Upper gastrointestinal endoscopy and colonoscopy were negative. Abdomen, chest and brain CT scans were negative. Bone scan was negative. These findings were consistent with primary malignant melanoma of urinary bladder. After thorough review of published scientific literature, we suggested radical cystectomy as the therapeutic option. The patient declined any further treatment so was given only symptomatic \& supportive treatment. Nine months later, she presented with hematuria and back pain. PET-CT showed increased uptake in lumbosacral vertebrae and liver, suggestive of metastases. She was advised palliative radiotherapy to the painful bone metastases and continued to receive palliative care. She succumbed to complications caused by widespread metastases fourteen months after the diagnosis.

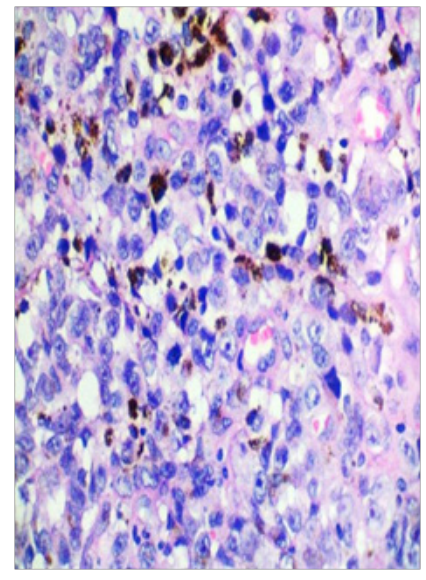

Figure I Hematoxylin and eosin, x45X; malignant melanocytes with melanin pigment.

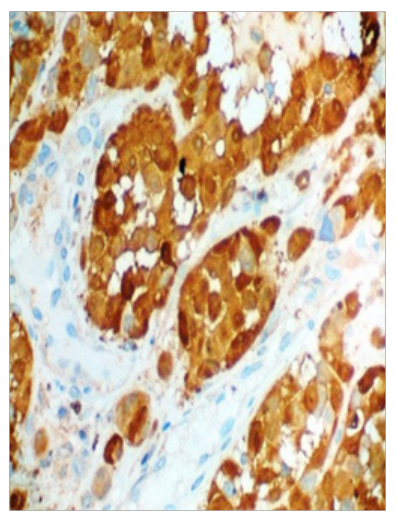

Figure 2 S-100: Cytoplasmic positivity in malignant melanocytes, (x45X). 


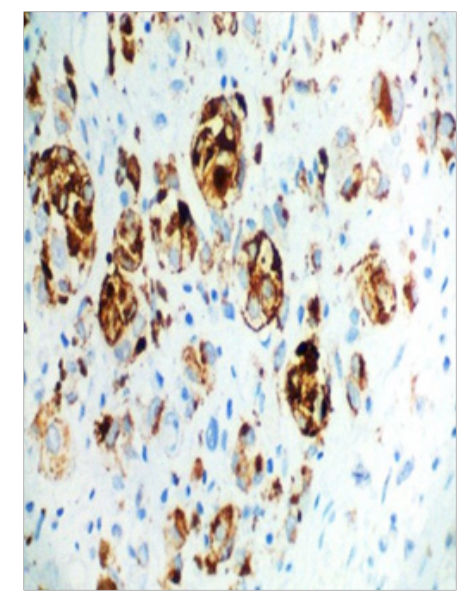

Figure 3 HMB-45: Cytoplasmic positivity in malignant melanocytes, (x45X).

\section{Discussion}

Primary malignant melanoma of urinary bladder occurs very rarely in urinary tract, especially in urinary bladder. ${ }^{3}$ Metastases of malignant melanoma to genitourinary tract is common and patients dying of melanoma have kidney (45\%) \& bladder (18\%) metastases. ${ }^{4}$ Therefore it is important to distinguish primary from a secondary melanoma in urinary bladder. Stein \& Ainsworth postulate ${ }^{5}$ the following criteria for considering a bladder melanoma a primary lesion:

i. No history of previous cutaneous lesion;

ii. No evidence of regressed cutaneous malignant melanoma;

iii. No evidence of other visceral primary melanoma;

iv. Pattern of recurrence should be consistent with the region of initial malignant melanoma;

v. Margins of bladder lesion should contain atypical melanocytes, Criterion

Regarding histological diagnosis, the microscopic examination of malignant melanoma reveals spindle or epitheloid cells. Epitheloid cells have marked pleomorphism, abundant cytoplasm, and prominent eosinophilic nucleoli. Cytoplasmic melanin pigment can be seen. Often atypical melanocytes are seen.

Clinical examination was negative for cutaneous lesion and there was no history or evidence of regressed melanomas. CT scans of abdomen, chest $\&$ brain at diagnosis were essentially normal. The immunohistochemical markers HMB-45 antigen \& S-100 protein became important components of diagnosis. ${ }^{6}$ In our case, HMB-45 and S-100 immunostaining were positive for tumor cells.
A number of treatment options are mentioned in literature for primary melanomas of urinary bladder. They include transurethral resection, partial cystectomy and total cystectomy with urinary diversion, chemotherapy, radiation therapy, immunotherapy with interferon-alpha and intravesical instillation of BCG. The prognosis however remains poor. ${ }^{78}$ Majority of patients died due to widespread metastases three years after initial diagnosis. Our patient received only palliative treatment and survived for 14 months.

\section{Conclusion}

Malignant melanoma of bladder is rare. Criteria reported by Ainsworth $^{5}$ are the most acceptable guidelines to confirm the diagnosis. A careful review of histological features and performing immunohistochemical-staining procedures for S-100 and HMB-45 are very important in confirming the diagnosis. Due to rarity of the tumor no standard treatment guidelines are available. Most of the patients in literature were treated as melanomas of other commoner sites are treated.

\section{Acknowledgements}

None.

\section{Conflict of interest}

The author declares no conflict of interest.

\section{References}

1. Stein BS, Kendall AR. Malignant melanoma of the genitourinary tract. $J$ Urol. 1984;132(5):859-868.

2. Venyo AK. Melanoma of the urinary bladder: a review of the literature. Surg Res Pract. 2014;605802.

3. Tainio HM, Kylmala TM, Haaspalo HK. Primary malignant melanoma of the urinary bladder associated with widespread metastases. Scand J Urol Nephrol. 1999;33(6):406-407.

4. Sayar H, Erdogan S, Adamhasan F, et al. Malignant melanoma of the bladder: A case report. Can Urol Assoc J. 2014;8(1-2):E54-56.

5. Ainsworth AM, Clark WH, Mastrangelo M, et al. Primary malignant melanoma of the urinary bladder. Cancer. 1976;37(44):1928-1936.

6. Xu X, Chu AY, Pasha TL, et al. Immunoprofile of MITF, tyrosinase, melan-A, and MAGE-1 in HMB45-negative melanomas. Am J Surg Pathol. 2002;26(1):82-87.

7. Pacella M, Gallo F, Gastaldi C, et al. Primary malignant melanoma of the bladder. Int J Urol. 2006;13(5):635-637.

8. Van Ahlen H, Nicolas V, et al. Primary melanoma of urinary bladder. Urology. 1992;40(6):550-554. 